\title{
STATUS OF THE RF SYSTEM FOR THE ANKA STORAGE RING
}

\author{
D. Einfeld, F. Pérez ${ }^{+,+}$, S. Voigt, ANKA, Forschungszentrum Karlsruhe, Germany \\ A. Fabris, C. Pasotti, M. Svandrlik, Sincrotrone Trieste, Italy
}

\section{Abstract}

The RF system for the ANKA storage ring has two plants, each plant consists of one $250 \mathrm{~kW}$ transmitter at $500 \mathrm{MHz}$ feeding two cavities, with all the peripherals. The different components, namely the high voltage power supply, the klystron, the waveguide system and the cavities, are now under construction and test. The HV power supply is provided by Jäger (Germany), and is been built on site. The first klystron, from the company EEV (England), has been assembled at the factory and the next two are under way. The components of the waveguide system have been already delivered by the firm Dielectric (USA), except the circulator that has been built by AFT (Germany). The factory acceptance tests of the cavities, together with the low level electronic systems, consisting of a frequency loop for each cavity and an amplitude and phase loop for each RF plant, have started at the end of 98 and, following the foreseen schedule, will be concluded in July 99 at Sincrotrone Trieste (Italy).

\section{INTRODUCTION}

During the present year the storage ring ANKA is being constructed at the Forschungszentrum Karlsruhe. It will be completely assembled after summer and the commissioning should start in October of this year [1].

The ANKA storage ring is designed to run at an energy of $2.5 \mathrm{GeV}$ and at a maximum beam current of $400 \mathrm{~mA}$.

Two RF plants, each one with two cavities powered by a single $250 \mathrm{~kW}$ klystron, will be installed. Figure 1 shows a 3D view of one of these plants and in table 1 the main parameters are listed.

At the moment, most of the components are in the latest stage of construction and some of them have been already tested at the factory. The waveguide system has been delivered two weeks ago and the rest of components will arrive within the next four months.

\footnotetext{
${ }^{+}$On leave from LSB-IFAE, Barcelona, Spain

${ }^{++}$Email: perez@anka.fzk.de
}

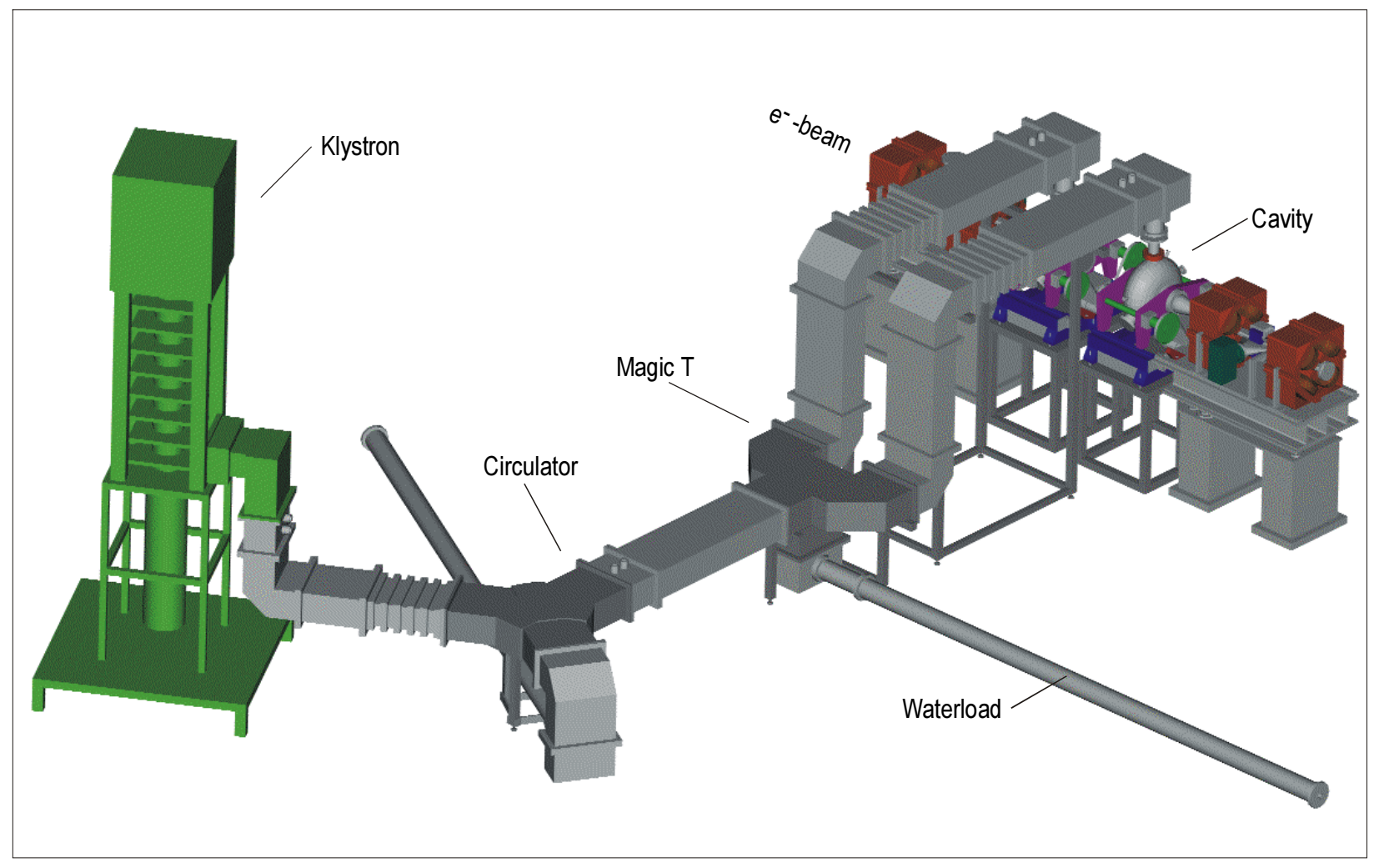

Figure 1: 3D view of one RF plant 
Table 1: Main RF parameters

\begin{tabular}{|l|c|}
\hline Parameter & Value \\
\hline \hline Beam Energy & $2.5 \mathrm{GeV}$ \\
\hline Energy Loss per Turn & $662 \mathrm{keV}$ \\
\hline Beam Current (maximum) & $400 \mathrm{~mA}$ \\
\hline Harmonic Number & 184 \\
\hline Revolution Frequency & $2.72 \mathrm{MHz}$ \\
\hline RF Frequency & $499.65 \mathrm{MHz}$ \\
\hline Momentum Compaction Factor & 0.0081 \\
\hline Energy Spread & $0.09 \%$ \\
\hline Total RF voltage & $2 \mathrm{MV}$ \\
\hline Overvoltage Factor & 3 \\
\hline Energy Acceptance & $1.5 \%$ \\
\hline Synchrotron Frequency & $36 \mathrm{kHz}$ \\
\hline Synchronous Phase & $160.7^{\circ}$ \\
\hline Bunch Length & $9.8 \mathrm{~mm}$ \\
\hline Number of Cavities & 4 \\
\hline
\end{tabular}

\section{CAVITIES}

The cavities together with the low level electronics phase, amplitude and tuning loops- are provided by Sincrotrone Trieste, Italy.

\subsection{Cavity Parameters}

The two first cavities are ready and their main parameters have been measured. Table 2 compares the measured values with the target ones.

Table 2. Cavity parameters

\begin{tabular}{|l|c|c|c|}
\hline Parameter & Target & \multicolumn{2}{|c|}{ Measured } \\
\hline & & Cav 1 & Cav 2 \\
\hline \hline Resonant frequency, MHz & 499.65 & 499.64 & 499.65 \\
\hline Quality factor & 40000 & 38900 & 41000 \\
\hline R/Q & 175 & 174 & 174 \\
\hline Shunt Impedance, $\mathrm{M} \Omega$ & 3.3 & 3.3 & 3.5 \\
\hline Max Coupling Coefficient & 3.5 & 3.3 & 3.6 \\
\hline
\end{tabular}

The lower quality factor of the first cavity is due to a contamination of the cavity's surface by the filler material used during the fabrication procedure. The problem has been fixed and solved as can be seen by the higher quality factor of the second cavity. The next two cavities are expected to have parameters similar to those of the second one.

The HOMs of both cavities have been already characterised. The results are presented in a separate paper in this conference [2]

\subsection{High Power Test}

Two different power tests have been performed with the first cavity:
- Forward power test: 8 hours of continuously running at $52 \mathrm{~kW}$ CW with the cavity on tune, figure 2.

- Reflected power test: $1 / 2$ hour of continuously running at $52 \mathrm{~kW} \mathrm{CW}$ with the cavity out of tune. This test is done mainly to test the input coupler.

Both tests have been successfully passed at the power stand test of Sincrotrone Trieste, figure 3.

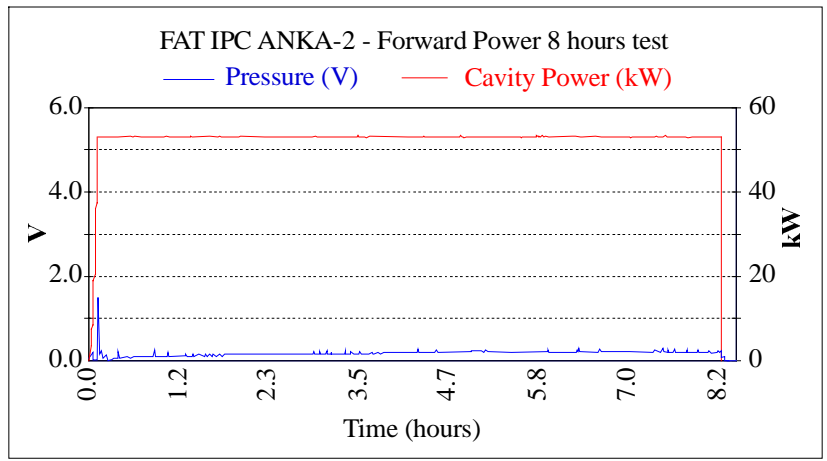

Figure 2.8 hours power test without vacuum trips

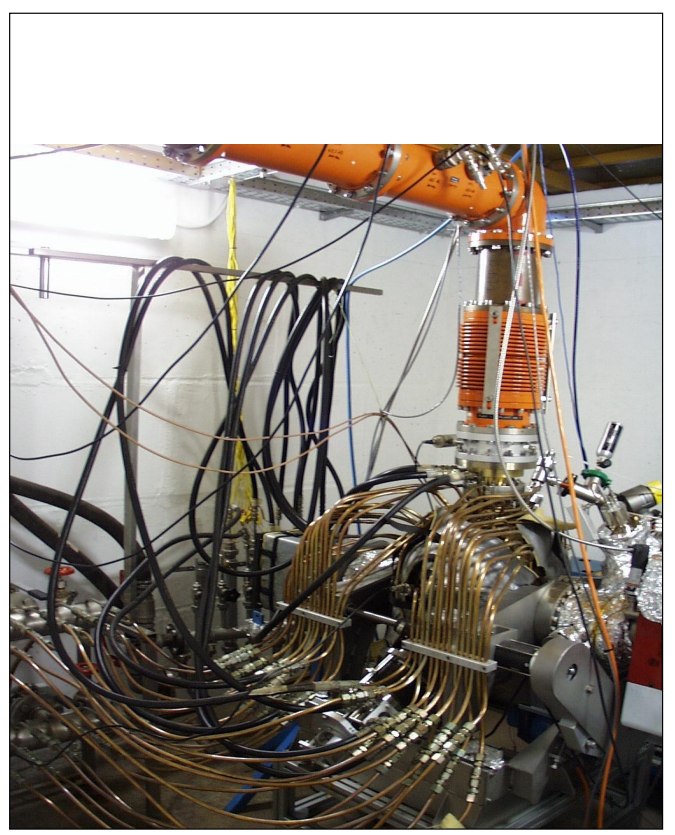

Figure 3. Cavity at the power stand during the power test

The power test of the second cavity will be done at the beginning of April, the third at the end of Mai and the last one at the beginning of July.

\section{LOW LEVEL ELECTRONICS}

The low level electronics for the ANKA RF system will be composed of four frequency loops (one for each cavity), two amplitude and two phase loops (one for each RF plant) plus all the ancillary equipments for the phasing of the two plants and the interlock electronics [3]. The design of the low level electronics has taken into account the different operating conditions of ANKA, where the beam is injected at $500 \mathrm{MeV}$ and ramped in the ring to the final $2.5 \mathrm{GeV}$ energy. Amplitude and phase of the cavity 
gap voltages must be kept constant during the different status of machine operation (injection, ramping and beam storage), considering that all the low level electronics loops interact with each other and are coupled via the beam.

From the construction point of view, the low level electronics has been designed in a modular way which eases maintenance and troubleshooting. All the low level electronics for the ANKA RF system will be hosted in six racks, three for each plant.

The first frequency loop has been tested together with the first cavity, satisfying the required specifications. Table 3 compares the target and measured parameters.

Table 3. Frequency loop parameters

\begin{tabular}{|l|l|l|}
\hline Parameter & Target & Measured \\
\hline \hline Speed (nominal), Hz/sec & 700 & 704 \\
\hline Speed (maximum), Hz/sec & 1000 & 1040 \\
\hline Op. Loop 3 dB bandwidth, Hz & 200 & 210 \\
\hline $100 \mathrm{~Hz}$ option & & \\
\hline Sensitivity of the loop, Hz & 100 & $75-100$ \\
\hline Open loop dc gain, dB & $>36$ & 41 \\
\hline $500 \mathrm{~Hz}$ option & & \\
\hline Sensitivity of the loop, Hz & 500 & 450 \\
\hline Open loop dc gain, dB & $>23$ & 28 \\
\hline
\end{tabular}

The second frequency loop, the first amplitude and phase loops and ancillary equipments will be tested at the beginning of next April, together with the second storage ring cavity. Therefore at this stage all the low level electronics for one of the two storage ring plants will be completed. The low level electronics for the second plant is now under assembly. Each component has been acquired and individually tested. The acceptance test of the low level electronics for the second RF plant is planned in parallel with the factory acceptance tests of the third and fourth storage ring cavities.

\section{TRANSMITTER}

The transmitter will be assembled at ANKA and it is formed by a $250 \mathrm{~kW}$ klystron, a $470 \mathrm{kVA}$ high voltage power supply, a PLC system for its control and interlock, and the ancillaries components to complete it: power supplies, sensors, etc.

The klystrons are produced by EEV, at England. The tube of the first klystron has been already assembled and is now under vacuum conditioning. Three klystrons will be delivered, two for operation of the machine and one as a spare. The main parameters are listed in table 4 .

Table 4. Main parameters of the klystron

\begin{tabular}{|l|c|}
\hline Parameter & Value \\
\hline \hline Operating frequency & $499.65 \mathrm{MHZ}$ \\
\hline RF output power & $>250 \mathrm{~kW}$ \\
\hline Efficiency & $>62.5 \%$ \\
\hline
\end{tabular}

The high voltage power supply is at the moment being assembled at ANKA by the company JÄGER, from Germany. Its main parameters are listed in table 5.

Table 5. Main parameters of the HV power supply

\begin{tabular}{|l|c|}
\hline Parameter & Value \\
\hline \hline Voltage & -20 to $-52 \mathrm{kV}$ \\
\hline Current & $9 \mathrm{~A}$ \\
\hline Stability & $\pm 0.5 \%$ \\
\hline Ripple, peak at peak & $<0.4 \%$ \\
\hline Maximum stored energy & $20 \mathrm{Ws}$ \\
\hline
\end{tabular}

For the control and interlock of the transmitter we have chosen to use an industrial PLC standard from the company BOSCH. This is separately presented in this conference [4].

The rest of the components, power supplies for the focusing coils and the gun of the klystron, sensors and connectors, etc are already in house.

The assembly of the first transmitter will start at the end of April, when all the components will be delivered, and during May it will be brought into operation.

\section{WAVEGUIDE SYSTEM}

The waveguide system has been designed to feed two cavities with only one klystron. A circulator to isolate the klystron and a magic tee to split the power in two are the main components (figure 1).

The distance from the centre of one cavity to the other one is $900 \mathrm{~mm}$, i.e. one and a half the wavelength of the $500 \mathrm{MHz} \mathrm{RF}$ voltage. For that reason the power input loop of one cavity will be rotated $180^{\circ}$ with respect the position of the loop at the other cavity, in that way the bunches will see the correct phase. The difference in phase between the two arms of the magic tee has been measured at Dielectric and it is less than $1^{\circ}$. It has to be noted that no phase shifter has been foreseen, so that we rely on the symmetry and the proper performance of the components.

\section{SUMMARY}

The first RF plant with two cavities will be ready in June and the second plant in August.

\section{REFERENCES}

[1] D.Einfeld, S.Hermle, E.Huttel, A.Kruessel, M.Lange, F.Pérez, M.Pont, U.Ristau, R.Rossmanith, H.Schieler, S.Voigt, R.Walther, A.Weindl, M.Plesko, "Status of the accelerator for the $2.5 \mathrm{GeV}$ light source ANKA in Karlsruhe", these proceedings.

[2] A.Fabris, C.Pasotti, M.Svandrlik, "Characterization of the ANKA RF Cavities HOMs for Coupled Bunch Instability Calculations", these proceedings.

[3] A.Fabris, C.Pasotti, M.Svandrlik, D.Einfeld, F.Pérez, S.Voigt, "Low level RF System for the ANKA Storage Ring", EPAC 98, p1761.

[4] D.Einfeld, F.Pérez, S.Voigt, M.Humpert, "Interlock and Control for the RF System of the ANKA Storage Ring", these proceedings. 